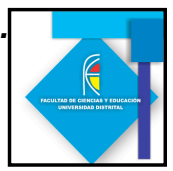

\title{
EL PÁJARO BEBEDOR COMO HERRAMIENTA PARA GENERAR APRENDIZAJE POR CONTRADICCIÓN
}

\author{
DRINKER BIRD AS A TOOL TO CREATE LEARNING BY \\ CONTRADICTION
}

\author{
Diego Fernando Vera Valcárcel ${ }^{1}$ \\ Diego Julián Rodríguez ${ }^{2}$
}

\section{Resumen}

Muchos científicos han intentado violar la segunda ley de la termodinámica a partir de la construcción de una maquina que ha sido denominada de movimiento perpetuo. Una maquina hipotética que seria capaz de continuar funcionando eternamente, después de un impulso inicial, sin necesidad de energía externa adicional, teóricamente imposible de construir. Teniendo en cuenta la necesidad que existe en despertar el espíritu científico en los estudiantes, nace la posibilidad de llevar una maquina hipotética de movimiento perpetuo didáctica y divertida que genera interés en aprender de los fenómenos y conceptos físicos, llevándolos al aula para percibir lo que ocurriría sí estos no se cumplieran, a través de la formulación de preguntas como: ¿Qué pasaría si no hubiera gravedad? ¿Flotaríamos? Y generando así aprendizaje por contradicción. Por tal razón se diseñó una máquina de movimiento perpetuo que posibilitara esta contradicción, el "Drinking Bird" o pájaro bebedor resulta ser una maquina cercana a generar este movimiento y simularía de manera adecuada lo que ocurriría si no se cumpliera la segunda ley de la termodinámica llevando a comprender el fenómeno con mayor claridad, para finalmente comprobar su principio a partir de un experimento real que contradiga su definición.

Palabra clave: Segunda ley de la termodinámica, maquina de movimiento perpetuo, aprendizaje, contradicción, pájaro bebedor.

\begin{abstract}
\footnotetext{
${ }^{1}$ Universidad Pedagógica Nacional, diegomero10@hotmail.com
}

2 Universidad Pedagógica Nacional, djrodriguezp@gmail.com
\end{abstract}

Many scientists have tried to violate the second law of thermodynamics from building a machine that has been called perpetual motion. A hypothetical machine would be able to 


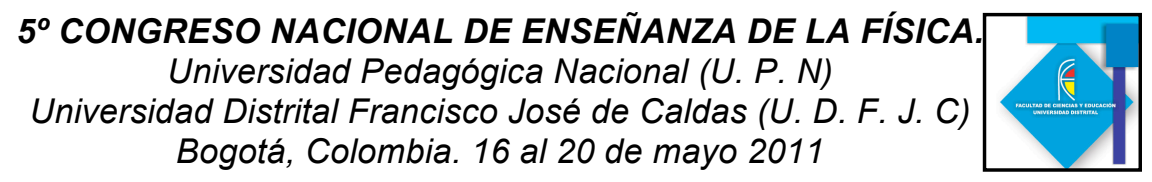

continue working forever, after an initial boost, without additional external power, theoretically impossible to build. Given the need in awakening the scientific spirit among students, created the possibility of a perpetual motion machine hypothetical educational and fun that generates interest in learning about physical phenomena and concepts, bringing them into the classroom to perceive what would happen if these are not met, through asking questions like: What if there were no gravity? "Float around? Learning and generating contradiction. For this reason we designed a perpetual motion machine that enabled this contradiction, the "Drinking Bird" or bird drinker turns out to be a machine close to generating this movement properly simulate what would happen if you do not fulfill the second law of thermodynamics leading to understand the phenomenon more clearly, to finally see their debut from a real experiment that contradicts its definition.

Keywords: Second law of thermodynamics, perpetual motion machine, learning, contradiction.

\section{Introducción}

De un interés por la segunda ley de la termodinámica e indagando el fundamento de su principio, se abordaron un sinfín de documentos que hablan del movimiento perpetuo, resulto apropiado articular el trabajo experimental con este concepto, por tal razón es pertinente señalar que desde la educación media se ha explicado la física a partir de situaciones experimentales, lo que hacemos es intentar refutar la ley para comprobar que es cierta, pero en el caso de la segunda ley de la termodinámica ha resultado un poco más complicado, ya que se ha construido una maquina que nos da la posibilidad de pensar en un movimiento perpetuo, la posibilidad de llevar a la escuela la segunda ley de la termodinámica comprobando su principio a partir de un experimento mental que plantea lo que ocurriría si esta no se cumpliera, si esta fuera falsa, contradiciendo su definición. El diario montañés de Santander (España) publico un articulo llamado "La física detrás del pájaro bebedor", que señala las propiedades físicas de este artefacto, qué hasta la fecha está siendo utilizado solamente como un juguete. (Gutiérrez, 2009)

Desde la época del renacimiento se ha intentado refutar la segunda ley de la termodinámica en el mundo científico. Muchos pensadores en infinidad de campos lo han pensado ,Científicos como Maxwell con su máquina llamada "El demonio de Maxwell", Richard Feynman con "El trinquete de Brown" o Robert Boyle con su "frasco de auto llenado" lo han intentado, pero no lo han logrado; lo más cerca que han estado es la construcción de una máquina que ha sido denominada de movimiento perpetuo, una máquina hipotética que sería capaz de continuar funcionando eternamente, después de un impulso inicial, sin necesidad de energía externa adicional, teóricamente imposible de construir , ya que violaría la segunda ley de la termodinámica .

En la búsqueda de esta hipotética maquina se ha encontrado una que resulta muy didáctica y divertida, el "pájaro bebedor" o (drinking bird). Este dispositivo se mueve, se 


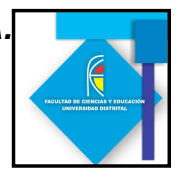

balancea periódicamente, sin partes móviles aparentes y sin un origen claro de la fuente de energía que lo mantiene en movimiento.

A simple vista se puede llegar a decir que en algún momento esta propuesta de investigación tiene como objetivo refutar el postulado que define a la segunda ley de la termodinámica, pero no es así, el objetivo fundamental es proporcional al estudiante una herramienta de aprendizaje distintas a las que se han utilizado habitualmente. Qué mejor manera de capturar su curiosidad que con la creación de un artefacto didáctico que además de ser sencillo y divertido posea propiedades físicas tan impresionantes que les despierte el interés por aprender termodinámica, no de la manera habitual, sino intentando entender un fenómeno o concepto apartrir de un instrumento que sea capaz de simular lo que ocurriría si el postulado que define este concepto o fenómeno no se cumpliera.

\section{Material y método}

En un comienzo la intención era buscar un artefacto qué genera interés y motivación en los jóvenes por aprender de la segunda ley de la termodinámica además de que fuera adecuado para el desarrollo de un trabajo experimental, en esta búsqueda se encontraron diversos modelos que eran de gran ayuda pero ninguno resulto ser tan apropiado y llamativo para los alumnos como el pájaro bebedor o "drinking bird", resulta extraño pensar que jóvenes a nivel universitario pudieran llegar a interesarse por este tipo de "juguete", que a simple vista es solo eso, un juguete, pero al detallar su funcionamiento se descubren un a serie de propiedades físicas que lo diferencian de cualquier.(figura1)

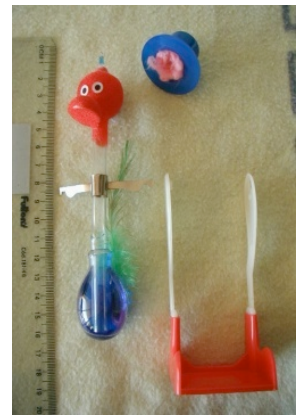

Figura1: Esquema pájaro bebedor

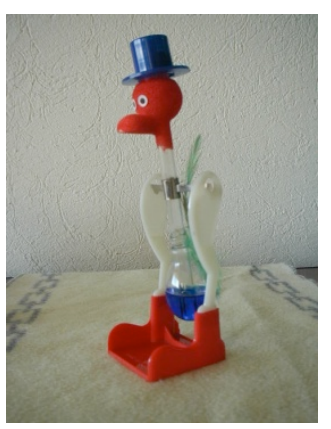

Figura 2: Esquema partes pájaro

El pájaro está formado por una especie de esfera inferior ("cuerpo") de vidrio, y otra superior ("cabeza"), comunicada por un tubo ("cuello") también de vidrio. Este conducto llega hasta el borde interior de la cabeza (sin entrar) pero penetra casi hasta el fondo del interior del cuerpo. (Figura 2)

Como se ve en la (figura 1) donde el pájaro está casi vertical, el interior del cuerpo está parcialmente ocupado (un $60 \%$ aprox.) por un líquido con colorante, que separa el aire encerrado en el cuerpo, del aire encerrado en el cuello y la cabeza. 


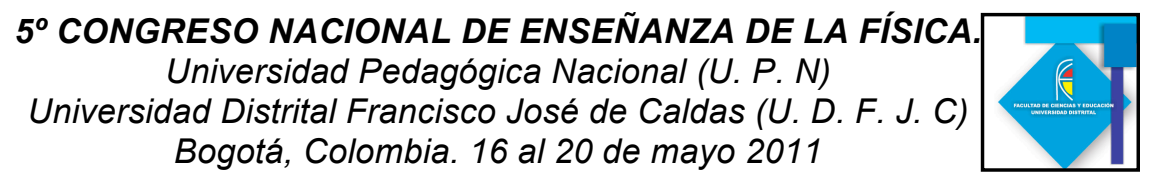

Cuando la cabeza del pajarito se mete al agua, la cabeza del pajarito se empapa (tiene una esponja), al mismo tiempo que debido a que en ese momento el pajarito está en posición horizontal, entran en contacto más moléculas del vapor con el líquido. La cola es más grande y contiene más líquido que la cabeza por lo que el centro de gravedad está desplazado hacia este extremo, lo cual hace que el pajarito se ponga solo en posición vertical.

La cabeza, que ahora está mojada se enfría por la evaporación del agua. En los gases, a volumen constante, la presión y la temperatura son variables muy sensibles (ley de GayLussac). La presión del vapor cerca de la cabeza disminuye, lo que hace que la columna del líquido suba hacia la cabeza, haciendo la función de un péndulo haciendo oscilar al pajarito. El movimiento del péndulo va disminuyendo con el tiempo hasta que hay suficiente líquido cerca de la cabeza para que se repita el ciclo al ponerse el pajarito horizontal y absorber más agua con la esponja.

\section{iLa física del pájaro bebedor!}

No existe ninguna máquina térmica que funcione entre dos focos térmicos a distinta temperatura cuyo rendimiento sea mejor que el de una máquina de Carnot entre esos mismos focos (Figura 3). El pájaro bebedor resulta eficaz para explicar el funcionamiento de una máquina térmica (aunque no es un ciclo de Carnot) esto si ocurre en el interior del pájaro bebedor. Por tal razón resulta adecuado a partir de la siguiente analogía explicar su funcionamiento: $\mathrm{Si}$ tenemos un gas encerrado en un pistón que se calienta a una temperatura $(T)$. Se puede ver una expansión isotérmica en el ciclo 1-2, el gas que se encuentra ocupando el mínimo volumen al entrar en contacto con la fuente de calor, lo absorbe pero no cambia su temperatura, el gas utiliza esa energía para aumentar su volumen. Cuando el ciclo 1-2 ya no necesita absorber más calor su comportamiento se describe en el ciclo 2-3. Como no hay calor aportado desde el exterior, el gas se enfría al expandirse y ocupa el volumen máximo posible. En el ciclo 3-4 se pone el foco a temperatura T2 (T2 es mayor que T1) y el gas se empieza a comprimir. No aumenta su temperatura porque cede calor al foco térmico que está a menor temperatura. Una vez se retira el foco y el sistema vuelve a estar aislado se cierra el ciclo con la compresión adiabática 4-1 en la que el sistema se comprime y recupera el estado inicial. (Figura 4). (MIGUI, 2009)

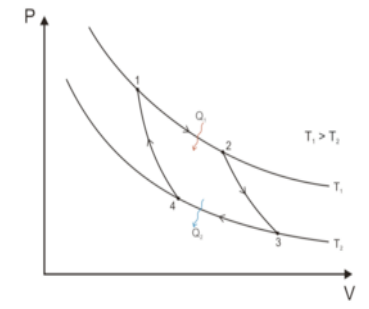

Figura 3: Grafica pájaro bebedor

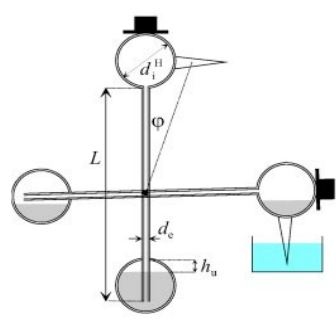

Figura 4: Esquema fun. Pájaro bebedor 


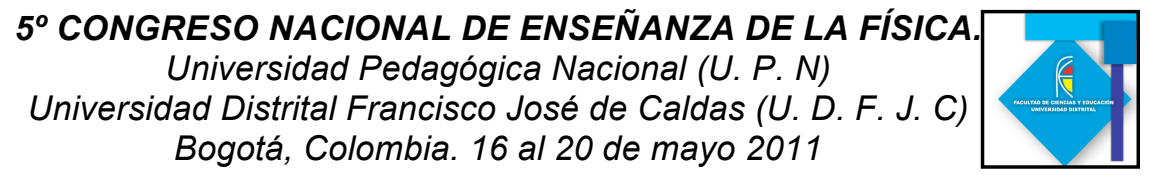

Es de gran importancia encontrara la relación existente entre este dispositivo y la segunda ley de la termodinámica, como sabemos esta ley posee dos enunciados importantes:

Es imposible para cualquier dispositivo que funcione en un ciclo recibir calor de una sola fuente y producir una cantidad neta de trabajo [Enunciado de Kelvin-Planck para la Segunda Ley].

Es imposible construir un dispositivo que funcione en un ciclo y cuyo único efecto sea producir la transferencia de calor de un cuerpo de temperatura más baja a un cuerpo de temperatura más alta. [Enunciado de Causías para la Segunda Ley].

Pero a partir de estos dos postulados se desprenden otros enunciados a los que se llega por simple conclusión, los que se denominan corolarios o enunciados alternativos. Un corolario es una afirmación lógica que es consecuencia inmediata de un teorema. En esta investigación nos centraremos en el siguiente:

Toda maquina o sistema en movimiento terminara por detenerse ya que existe una perdida de energía que no se puede recuperar. Así todo en el universo esta sujeto a al muerte por enfriamiento, por desgaste entre otros.

Actualmente se esta llevando acabo la fase de implementación en donde los estudiantes diseñaran una maquina de movimiento perpetuo, el pájaro bebedor o "Drinking Bird", para que se den cuenta de su funcionamiento y lleguen de manera intuitiva a la definición real de la segunda ley de la termodinámica a partir lo que ocurriría si esta no se cumpliera, relizando el calculo matemático y la interpretación física de su funcionamiento. Generando en ellos aprendizaje por contradicción y despertando el espíritu científico que resulta ser fundamental para el futuro de las ciencias.

\section{Referencias Bibliográficas}

Giordano, José. (2005).Internet, Cómo funciona el pájaro bebedor. Extraído el 15 de septiembre, 2010.9:30 pm. http://www.worldlingo.com/ma/enwiki/es/Perpetual_motion

Gutiérrez, Julio. (2009).Internet, LA física detrás del pájaro bebedor. Revista montañés. Extraído el 04 de septiembre, 2010. 5:00 pm. http://blogs.eldiariomontanes.es/scientiamater/2009/6/18/la-fisica-detras-del-pajaro-bebedor

Holman, J.P. (1975).Termodinamica.Mexico: McGraw-Hill

Migui, ciencia y tecnología. (2009).Internet, Procesos adiabáticos en la vida cotidiana. Extraído el 18 de marzo del 2010. http://www.migui.com/ciencias/fisica/procesosadiabaticos-en-la-vida-cotidiana.html 